\title{
The thoracic oncology specialist: curriculum recommendations in thoracic oncology training
}

\author{
Anne-Pascale Meert ${ }^{1}$, Julie-Lyn Noël ${ }^{2}$ and Fernando Gamarra ${ }^{3,4}$ on behalf of \\ the Thoracic Oncology HERMES Task Force
}

Affiliations: 'Service des Soins Intensifs et Urgences Oncologiques, Oncologie Thoracique, Institut Jules Bordet, Brussels, Belgium. ${ }^{2}$ Educational Activities Dept, European Respiratory Society, Lausanne, Switzerland. ${ }^{3}$ Division of Respiratory Medicine, Klinikum Straubing GmbH, Straubing, Germany. ${ }^{4}$ For a list of the Thoracic Oncology HERMES Task Force members and their affiliations see the Acknowledgements section.

Correspondence: Anne-Pascale Meert, Service des Soins Intensifs et Urgences Oncologiques, Oncologie Thoracique, Institut Jules Bordet, 1 rue Heger Bordet, Brussels 1000, Belgium. E-mail: ap.meertabordet.be

@ERSpublications

HERMES proposes a detailed, interprofessional and multidisciplinary curriculum for training in thoracic oncology http://ow.ly/H1A9301jVpx

The power of one, if fearless and focused, is formidable, but the power of many working together is better.

Gloria Macapagal Arroyo

Thoracic oncology is a rising discipline that is evolving towards being a specialised multidisciplinary activity. Multidisciplinary teams (MDTs) are playing an ever important and increasing role in the management of thoracic malignancies in the lung, pleura, chest wall and mediastinum. These teams have a great potential to improve patient care and the healthcare system [1]. A thoracic oncology MDT denotes a group of healthcare specialists from different backgrounds and expertise providing efficient and comprehensive care for patients suffering from thoracic malignancies.

Among thoracic malignancies, lung cancer has a significant worldwide impact because it is estimated to cause 1.59 million deaths worldwide, as reported by the World Health Organization [2]. The epidemiology of this disease has evolved at an astounding rate such that, at present, it is considered a pandemic with major financial and social consequences. HARDAVELLA and SETHI [3] discussed challenging perspectives and future needs in lung cancer care, which include novel diagnostics and therapies anticipated to improve survival and outcomes, risk factors such as asbestos and smoking, lung cancer screening, epidemiological databases, changing trends of the disease, the late stage at presentation, and socioeconomic changes such as austerity measures in Europe. With these challenges, lung cancer care is generally characterised by a coordinated, multidisciplinary, process-oriented approach. As in lung cancer, the multidisciplinary approach would play a critical role in the management of thoracic malignancies as well. This is due to the increasing wealth of information, and the complexity of various medical procedures and interventions. In Europe, treatment is provided by physicians from different specialties, and is increasingly subject to regulations and quality control. In Belgium, for example, laws have been developed to regulate cancer care. Seven oncology-specific laws have been put in place, the first defining the multidisciplinary oncology consultation and allowing reimbursement for such care. Almost all innovative and expensive drugs are reimbursed only if all members of the MDT agree that they would benefit an individual patient [4].

Received: May 272016 | Accepted: June 022016

Conflict of interest: J-L. Noël is an employee of the European Respiratory Society. Further disclosures can be found alongside this article at erj.ersjournals.com

Copyright OERS 2016 
The European Respiratory Society (ERS) recognises the need to tackle these challenges and improve the quality of care received by those suffering from thoracic malignancies. The ERS Thoracic Oncology Assembly and the HERMES (Harmonising Education in Respiratory Medicine for European Specialists) initiative work together to develop educational standards. The Thoracic Oncology HERMES Task Force has recently published curriculum recommendations for training in thoracic oncology [5]. The curriculum recommendations further develops the syllabus published in 2013 [6]. The Task Force has proposed a detailed curriculum containing a sophisticated blend of educational strategies, content, learning outcomes, educational experiences, assessment and educational environment [7].

The Thoracic Oncology HERMES Task Force aims to address the training needs of the members of the MDT, any of whom can lead a thoracic oncology MDT team with appropriate training. Additional training will make these specialists more efficient in their interaction with the patients and fellow physicians. GAGA et al. [8] discussed the importance of high-volume specialised centres and MDTs, and advocated that treatment of patients with thoracic tumours should be concentrated where all the involved specialties are available. These specialists will need to be certified for their competence.

This curriculum details how to train to become a thoracic oncology specialist. According to the understanding of the Task Force, thoracic oncology specialists are expected to be well experienced clinicians who treat thoracic malignancies in a specialised centre. The target audience of this curriculum is respiratory physicians, medical oncologists, radiation oncologists and thoracic surgeons. Each of these specialists is familiar with the collaborating disciplines. This permits them to understand and appreciate the contribution of each. Thoracic oncology specialists are therefore in a position to lead a multidisciplinary thoracic oncology team and chair the tumour board.

The curriculum modules were formulated by the Task Force keeping in mind the concept of the thoracic oncology specialist. Table 1 shows the 22 curriculum modules.

The Thoracic Oncology HERMES Task Force was led by two chairs, Anne-Pascale Meert and Fernando Gamarra, and a medical education specialist, Julie-Lyn Noël, who coordinated the activities of the group. The other members were chosen to represent the treating medical disciplines involved in thoracic oncology: respiratory medicine, medical oncology, radiation oncology and thoracic surgery. Respiratory physicians and medical oncologists who were active members of the ERS included: Mina Gaga (Greece), Bogdan Dragos Grigoriu (Romania), Rudolf M. Huber (Germany), Sam Janes (UK) and Jean-Paul Sculier (Belgium). Georgia Hardavella (UK) represented the junior members of the ERS Thoracic Oncology Assembly. Furthermore, a pathologist, Philipp A. Schnabel (Germany), specialised in thoracic tumours is included. The Task Force also collaborated with other European Societies: Gilbert Massard (France), Dirk Van Raemdonck (Belgium) and Alessandro Brunelli (United Kingdom) participating as official representatives of ESTS (European Society of Thoracic Surgeons). Sara Ramella (Italy) and Paul Martin

TABLE 1 Thoracic oncology curriculum modules

1

2

3

4

5

6

7

8

9

10

11

12

13

14

15

16

17

18

19

20

21

22
General principles of the biology of thoracic cancers

Aetiology and epidemiology

Clinical presentations

Diagnostic procedures

Imaging

Diagnostic and interventional bronchoscopic techniques and medical thoracoscopy

Clinical and pathological staging

Pathology of intrathoracic tumours

Prognostic factors/predictive markers

Principles of thoracic surgery

Management of surgical complications

Principles of radiation therapy

Principles of systemic therapy

Side-effects of systemic therapy and their management

Combined-modality treatments

Management of particular groups of patients

Treatment evaluation and follow-up

Supportive care

Methodologies for clinical practice and research

Ethics

Cancer-related immunology

Quality and economic considerations in lung cancer treatment 
Putora (Switzerland) represented ESTRO (European Society of Thoracic Radiotherapy and Oncology); and Enriqueta Felip (Spain) and Anne-Marie C. Dingemans (the Netherlands) were chosen as medical oncology specialists by the ESMO (European Society for Medical Oncology). For the Task Force members' affiliations see the Acknowledgements section. In order to account for the different standards in Europe, the Task Force members were chosen to represent as many European regions as possible.

National respondents played an important role in the curriculum development. At the beginning for the syllabus development, they were expert respondents in the Delphi process, which was crucial for the content development. At this phase of curriculum development, they gave valuable feedback and validated the content. The national respondents consisted of experts nominated by the Forum of European Respiratory Societies in 2011 and the national coordinators of the European quality management in lung cancer care [9].

Contemporary concepts of medical education like Bloom's taxonomy of education objectives and Miller's model of clinical skills were applied $[10,11]$. Medical education specialists gave guidance on the concepts used. The curriculum is meant to be used as needed, and fit for the context of the trainee and trainer. The aim was to go beyond the mere list of topics and provide the learning outcomes, including the levels of complexity and assessment, in detail for each item. The curriculum gives concrete advice for the development of a training programme including the methods of assessment and the minimum clinical exposure times and patient numbers. With this information, trainees and trainers will be able to appraise what is needed and determine a unified education strategy to become a thoracic oncology specialist. The supplementary material to the curriculum on the Breathe website contains the complete module 22 (Quality and economic considerations in lung cancer treatment) [5].

Those who would complete the thoracic oncology training are already specialists in a discipline that is involved in the treatment of thoracic malignancies. Accordingly, the prerequisites to complete training would include being a specialist in a discipline that is involved in the diagnosis and treatment of thoracic tumours, and to have a recognised certificate in the country in which the learner is currently working. The Task Force recommends a minimum of 12 months of full-time training and a maximum of 24 months depending on the size of the institution or centre in which the training takes place. It is therefore a prerequisite to have an already recognised certificate in the country in which the applicant is currently working.

The question of including other medical specialties like pathology or palliative care was discussed. The Task Force agreed that medical and surgical specialists who are actively involved in the patient treatment are placed in a position to lead the tumour board or MDT meeting, and can undergo training in thoracic oncology. Because this may vary from country to country, a definite list of specialties was not made.

Training should be interprofessional, multidisciplinary, post-specialty and part of continuing professional development leading to an additional post-specialty certificate attesting to an advanced expertise. The delivery of this curriculum should be through a modular training programme where modules can be followed separately, grouped into tracks or all followed together [12]. In the case of this curriculum, a specialist, for example, a thoracic surgeon, could follow a specific group of modules, or track, as needed. This specialist will not need modules regarding thoracic surgery. The current curriculum will be used as a basis to define mandatory and optional modules as tracks for specific specialists as a next step for the Task Force. Furthermore, the curriculum could also be the basis for intersociety collaborations in the delivery of a modular training programme.

The Thoracic Oncology HERMES Task Force proposes a detailed, interprofessional and multidisciplinary curriculum for training in thoracic oncology. This has been created using current concepts of medical education. We look forward to the development of structured educational programmes based on these curriculum recommendations within the ERS, by other professional societies, by local centres and through intersociety collaborations. We hope that this curriculum will help to define the scope of an advanced expertise in thoracic oncology. Well-trained thoracic oncology specialists working in the MDT will deliver the best quality of care to those suffering thoracic malignancies.

\section{Acknowledgements}

The members of the Thoracic Oncology HERMES Task Force are Alessandro Brunelli (Dept of Thoracic Surgery, St James's University Hospital, Leeds, UK), Anne-Marie C. Dingemans (Maastricht University Medical Centre, Pulmonology, Maastricht, the Netherlands), Enriqueta Felip (Hospital Univer. Vall d'Hebron, Barcelona, Spain), Mina Gaga (Athens Chest Hospital, 7th Respiratory Medicine Dept, Athens, Greece), Bogdan Dragos Grigoriu (University of Medicine and Pharmacy Iasi, Regional Institute of Oncology Iasi, Iasi, Romania), Georgia Hardavella (Dept of Respiratory Medicine, King's College Hospital, and Dept of Thoracic Medicine, University College London, London, UK), Rudolf M. Huber (Division of Respiratory Medicine and Thoracic Oncology, University of Munich, and Thoracic Oncology Centre Munich, Member of the German Centre for Lung Research (DZL CPC-M), Munich, Germany), Samuel Janes (University College London, Centre for Respiratory Research, London, UK), Gilbert Massard (Dept of Thoracic Surgery, Hôpitaux Universitaires de Strasbourg, Strasbourg, France), Paul Martin Putora 
(Kantonsspital St Gallen, Dept of Radiation Oncology, St Gallen, Switzerland), Jean-Paul Sculier (Institut Jules Bordet, Intensive Care and Thoracic Oncology (ULB), Brussels, Belgium), Philipp A. Schnabel (Institut für Allgemeine und spezielle Pathologie, Universität des Saarlandes, Homburg/Saar, Germany), Sara Ramella (Campus Bio-Medico University, Radiation Oncology, Rome, Italy) and Dirk Van Raemdonck (Dept of Thoracic Surgery, University Hospital Leuven, Leuven, Belgium).

\section{References}

1 Jazieh AR, Al Hadab A, Howington J. Thoracic oncology multidisciplinary teams: between the promises and challenges. Ann Thorac Med 2008; 3: 34.

2 World Health Organization. Fact Sheet N0 297 - Cancer. www.who.int/mediacentre/factsheets/fs297/en/ Date last updated: February 15, 2015. Date last accessed: March 6, 2016.

3 Hardavella G, Sethi T. Epidemiology: development and perspectives. In: Dingemans AMC, Reck M, Westeel V, eds. Lung Cancer (ERS Monograph). Sheffield, European Respiratory Society, 2015.

4 State of Victoria, Department of Health. Multidisciplinary Cancer Care Literature Review. www.gha.net.au/ Uploadlibrary/411214604MultidisciplinaryCancerCareLitReviewFINAL.pdf Date last updated: 2012. Date last accessed: March 15, 2016.

5 Gamarra F, Noël J-L, Brunelli A, et al. Thoracic oncology HERMES: European curriculum recommendations for training in thoracic oncology. Breathe 2016; 12: 249-256.

6 Meert AP, Noël JL, Boffetta P, et al. Thoracic Oncology HERMES: a European syllabus towards a harmonised education and training of thoracic oncology specialists. Breathe 2013; 9: 381-392.

7 Harden RM. AMEE Guide No. 21: Curriculum mapping: a tool for transparent and authentic teaching and learning. Med Teach 2001; 23: 123-137.

8 Gaga M, Powell CA, Schraufnagel DE, et al. An official American Thoracic Society/European Respiratory Society statement: the role of the pulmonologist in the diagnosis and management of lung cancer. Am J Respir Crit Care Med 2013; 188: 503-507.

9 Blum TG, Rich A, Baldwin D, et al. The European initiative for quality management in lung cancer care. Eur Respir J 2014; 43: 1254-1277.

10 Bloom BS. Taxonomy of educational objectives; the classification of educational goals. 1st Edn. New York, Longmans Green, 1956.

11 Miller GE. The assessment of clinical skills/competence/performance. Acad Med 1990; 65: Suppl., S63-S67.

12 Swanwick T, ed. Understanding Medical Education: Evidence, Theory and Practice. Hoboken, John Wiley and Sons, 2011 